Received: March, 2016

Accepted: September, 2016

ISSN $2006-6996$

\title{
DETECTION OF MULTIDRUG RESISTANT TUBERCULOSIS (MDR-TB) AMONG RIFAMPICIN-RESISTANT TB PATIENTS USING LINE PROBE ASSAY (LPA) IN KANO, NIGERIA
}

\author{
${ }^{* 1}$ Aminu, A.I. and ${ }^{2}$ Tukur, A.D. \\ ${ }^{1}$ Department of Microbiology, Bayero University, Kano, Nigeria \\ ${ }^{2}$ National Tuberculosis (TB) Reference Laboratory North-West Zone, Aminu Kano Teaching Hospital, Kano, \\ Nigeria. \\ *Correspondence author: aishatuaminuibrahim@gmail.com, 080545033260
}

\section{ABSTRACT}

The Emergence of drug-resistant Mycobacterium tuberculosis strains especially multidrug resistant-TB (MDR-TB) and indeed extensively drug resistant TB (XDR-TB) is considered a real threat to achieving TB control. Thus, the WHO identified the need for accelerated access to rapid testing for rifampicin resistance to improve case detection in all patients with suspected MDR and $X D R-T B$. This study aimed at detecting MDR status of rifampicin-resistant $T B$ patients in north western part of Nigeria, as such information was found to be rare in this part of Nigeria. It was a descriptive cross-sectional study conducted at Aminu Kano Teaching Hospital, North-West TB reference Laboratory and purposive sampling technique was used in selecting patients for the study. Data generated was presented using descriptive statistics. Sputum samples were collected and processed according to standard mycobacteriological procedures. The presence of $M$. tuberculosis complex (MTBC) was confirmed using SD BIOLINE TB Ag MPT64 Rapid test. Genotypic assays were carried out on these samples by the GenoType $\mathbb{B}$ MTBDRplus molecular line prove assay (LPA). The results of the study revealed that of the 88 sputum samples collected from rifampicin resistant (confirmed by Genxpert from referring centres) TB patients, 74 (84.09\%) were males and 14 (15.9\%) were females. Most of them were aged 21-30 years and 31-40 years. Also, 86 of the sputum samples were confirmed as MTBC. Genotypic assays revealed that 48/88 (54.5\%) of the samples were identified as MDR-TB, 29 (32.9\%) were rifampicin mono-resistant cases while only 1(1.36\%) was resistant to isoniazid and 8 samples were found to be susceptible to both isoniazid and rifampicin. The pattern of genetic mutations revealed that rifampicin resistance due to the missing rhoB WT gene and the presence of rhoB MUT gene was seen in $77 / 78(98.7 \%)$ and $51 / 78(61.4 \%)$ of the rifampicin-resistance samples respectively and isoniazid resistance due to katG missing WT gene and katG MUT gene region was seen in 35/78 (44.9\%) of the samples. Among the MDR-TB cases rifampicin resistance was associated with rpoB WT gene and rpoB MUT gene in $100 \%$ and $62 \%$ of the isolates, and INH resistance was associated with katG WT gene and katG MUT gene in $70.8 \%$ and $72.9 \%$ of the isolates respectively. INH resistance in the MDR-TB cases was further associated with InhA WT and InhA MUT in 16.67\% and $4.17 \%$ of the isolates respectively. The study concludes by identifying that the reported MDR-TB rate of 54.5\% among rifampicin-resistant TB patients in the study was rather alarming and also identifies the need for expertize in the conduct and diagnosis of rifampicin-resistance status of TB patients using the GeneXpert techniques lack of which may be detrimental to the health of the patient.Thus, the study solicits for a much more committed TB control programmesincluding provision and training of manpower by the relevant agencies as a well as putting more efforts towards rapid and accurate diagnosis of TB patients, and proper treatment and management of the infected cases to minimize the spread and evolution of MDR-TB and indeed XDR-TB whose treatment is presented with more challenges.

Keywords: Rifampicin resistance, MDR-TB, LPA, North-Western zone, Nigeria.

\section{INTRODUCTION}

Tuberculosis (TB) a curable disease currently remains one of the most important infectious diseases not only in Nigeria but worldwide, with drug resistance and multidrug resistance (MDR) identified as the major factors affecting the TB control strategies. The World Health Organization (2013a) revealed that $3.7 \%$ of new tuberculosis (TB) patients in the world have multidrug-resistant strains (MDR-TB) and levels are much higher in those previously treated- about $20 \%$.The annual global MDR-TB burden is estimated at around 400000 cases (WHO, 2010), and Nigeria as at 2011 has an estimated MDR-TB burden of $>1000$ cases with only $1 \%$ of the cases enrolled on treatment (WHO, 2013a).

The WHO (2006) stated that although causes of MDRTB are microbial, clinical and programmatic, drugresistant TB is essentially a man-made phenomenon, mainly attributed to patience non-compliance, inadequate supply and inappropriate administration of drug regimens, and inadequacies to monitor treatment and most importantly the presence of poorly organized or TB control program. The appearance of extra multidrug resistance (XDR) as documented by many works further compounded 
BAJOPAS Volume 9 Number 2 December, 2016

the MDR nature of TB mainly due to the fact that treatment of patients with XDR-TB is challenging because of the lack of potent anti-TB drugs (Barnard et al., 2012).

The currently recommended treatment for new cases of drug-susceptible TB is a regimen of four-line drugs: Isoniazid (INH), Rifampicin (RMP), Ethambutol(ETM) and Pyrazimide(Barnard et al., 2012). Treatment for the multidrug-resistant $\mathrm{TB}$, defined as resistant to Isoniazid (INH) and Rifampicin (RMP) - the two most powerful anti-TB drugs- is longer, and requires more expensive and more toxic drugs. According to Barnard et al. (2012) XDR-TB is defined as resistance to at least INH, RMP, a fluoroquinolone, and one of three injectables (amikacin, kanamycin, or capreomycin).

Resistance-associated point mutations, deletions, or insertions have been described for all first-line drugs and for several second-line and newer drugs (ethionamide, fluoroquinolones, macrolides and nitroimidazopyrans)(Barnard et al., 2012).Barnard et al. (2012) revealed that INH resistance involves mutations in several genes such as $k a t G$, which encodes the activating enzyme, catalase, and other regulatory genes such as inhA, ahpC-oxyR, ndhand furA.RIF on the other hand specifically inhibited the elongation of full-length transcripts,subsequently, the rpoB locus from MTB was characterized and mutations conferring the resistant trait were identified(Rattan et al., 1998). It is interesting to note that monoresistance to INH is relatively common while monoresistance to RMP is quite rare (Barnard et al., 2012). In fact, nearly $90 \%$ of RMP-resistant strains are also INH-resistant. Therefore, resistance to RMP may be used as a surrogate marker for MDR-TB.

Nathasonet al. (2010) identified that, preventing initial infectionwith MDR tuberculosis and managing the treatmentof existing cases appropriately are the keysto containing the spread of this disease. Thus, rapid laboratory detection of MDR- and XDR-TB is critical, as late diagnosis could cause delays in treatment and serves as a major contributor to increased transmission of TB in the community and may compromise the efficacy of therapy (Barnard et al., 2012). In 2013 the World Health Organization in its policy statement indicated that effort should be made to reach the 3million new TB case that are missed every year by the health system $70 \%$ of which live India, South Africa, Bangladesh, Pakistan, china, DR Congo, Mozambique, Nigeria, Ethiopia, phliphines and Myanmar (WHO, 2013b) and especially bearing the fact that only $7 \%$ of the estimated MDR-TB cases are detected (WHO, 2010). Barnard et al. (2012) emphasizes the need for accelerated access to rapid testing for rifampicin resistance to improve case detection in all patients with suspected MDR and XDR$T B$ and to identify the pattern of genetic mutations associated with the resistant strains. Thus, case detection is one of most important factor that determines the successful implementation of TB control strategies or otherwise.

In view of the above, coupled with the fact that information on MDR status of TB patients is rare in Nigeria, this study was aimed at revealing the MDR profiles of rifampicin-resistant TB patients that reported to the National TB Reference Laboratory of Aminu Kano Teaching Hospital, Kano from the northwestern part of Nigeria using Line Prove Assay (LPA).

\section{MATERIALS AND METHODS}

The study was conducted at Aminu Kano Teaching Hospital, North West TB reference Laboratory, Kano, Nigeria after receiving ethical clearance from the Ethical Review Committee of the hospital (Appendix I). The study was a descriptive cross-sectional study and purposive sampling technique was used in selecting patients for the study, which included all TB patients that reported to the laboratory and were rifampicin resistant cases (confirmed by Genxpert) from their respective health centers across NorthWestern Zone of Nigeria. Furthermore, except for three, all the patients were originally diagnosed with TB and were treated with World Health Organization category I therapy (i.e. treatment with INH, RIF, PZA and EMB for 2 months, followed by treatment with INH and RIF or INH, RIF, and PZA for an additional 4 months). New-smear positive TB patients were excluded from the study. A total of 88 sputum samples were collected from the TB patients that were referred to the laboratory from September 2013 to October 2015 upon their consent. Data generated from the study was presented using descriptive statistics.

A total of three first-morning sputum specimens were collected from the patients and were subjected to pretreatment involving digestion, homogenization, decontamination and concentration in Biosafety Cabinet Level II (BSL II)using NALC-NaOH solution, $0.067 \mathrm{M}$ phosphate buffer ( $\mathrm{pH}$ 6.8) and buffered Saline (PBS) according to NTBLCP SOP Manual (2011) and NCCLS (2000). Sputum samples were also ZiehlNeelsen stained and acid fast bacilli were detected. All sputum samples collected, reagents and equipment used were handled according to standard mycobacteriological procedures as described by NTBLCP SOP Manual (2011) and NCCLS (2000)

The SD BIOLINE TB Ag MPT64 Rapid test was used to confirm the identity of $M$. tuberculosis complex (MTBC) contained in the samples according to manufacturer's specifications(SD Bioline Kit, Standard Diagnostics, Inc., Korea, 2015). It is a rapid immunochromatographic identification test for the $M$. tuberculosis complex (MTBC) that uses mouse monoclonal anti-MPT64 which has sensitivity and specificity of $98.6 \%$ and $100 \%$ respectively. The cassette was removed from the foil pouch and placed on a flat dry surface thus exposing the sample well. For each sample, one hundred microlitres $(100 \mu \mathrm{l})$ of the treated sputum sample was then added into the sample well. After 15minnutes of sample application, the appearance of two colour (purple) bands ("T" test band and " $\mathrm{C}$ " control band) within the result window was considered a positive result. Samples that were confirmed as being MTBC were then used for further analysis.

Genotypic assays were carried out on each of the confirmed MTBC samples in three separate rooms using the GenoType ${ }^{\circledR}$ MTBDRplus molecular line probe assay (LPA) according to the manufacturer's specification (HainLifescienceGmbH, Nehren, Germany, 2015) and WHO (2008) recommendations in three stages indicated below. 
Additionally DNA extraction was performed in the BSL III laboratory, master mix preparation in a second room, and PCR and hybridization were performed in a third laboratory.

\section{1- DNA EXTRACTION PROCEDURE WITH GENOL YSE}

DNA extraction procedure with genolyse was done according to manufacturer's specifications (HainLifescienceGmbH, Nehren, Germany,2015) and WHO (2008).Using a sterile graduated pipette $0.5 \mathrm{ml}$ $(500 \mu \mathrm{l})$ of the decontaminated sputum sample was transferred into microcentrifuge tube. This was done for all the samples, after which the tubes were closed and centrifuged for 15 minutes at $10000 \mathrm{xg}$. The supernatant was discarded and $100 \mu$ l lysis buffer (ALYS) was added and re-suspended by vortexing gently for $30 \mathrm{sec}$. The tubes were arranged in a floater inside the BSC II and incubated for 5minutes in a water bath at $95^{\circ} \mathrm{C}$. Then, $100 \mu \mathrm{l}$ Neutralisation buffer (A-NB) was added and vortexed for 30 seconds and the tubes were centrifuged at maximum speed $(10,000 \mathrm{xg})$. The heavier debris formed the pellet and the lighter DNA (free from impurities) was suspended in the supernatant which was transferred into clean labeled micro-centrifuge tubes for further use.

\section{2- PCR amplification of the extracted DNA}

The master mix preparation was done according to manufacturer's specification (HainLifescienceGmbH, Nehren, Germany, 2015) and WHO (2008). The master mix was made up of $10 \mu$ of the $A M-A$ and $35 \mu$ l of AM-B Reagent which was placed in a PCR tube labeled with sample number and mixed very well.This was prepared inside dead air box in a clean DNA free room. Then $5 \mu \mathrm{l}$ of each sample (containing the extracted DNA from above) was added to the corresponding tube containing the master mix and then mixed gently by pipetting up and down a few times. The PCR tubes were then placed in a 30 cycle $(10+20)$ thermal cycler program for amplification. After amplification the DNA contained in the amplicons were denatured in the TwinCubator $\AA$ which was pre-warmed to $45{ }^{\circ} \mathrm{C}$ and $20 \mu \mathrm{l}$ of denaturation solution $(\mathrm{NaOH})$ was added to each labeled well of the TwinCubator $\AA$ tray followed by the addition of $20 \mu$ l of the amplicons respectively. The mixture was mixed gently by pipetting up and down five times and then incubated at room temperature for 5 mins.

\section{3- Hybridization and detection}

Hybridization and detection procedures were carried out according to according manufacturer's specification (HainLifescienceGmbH, Nehren, Germany, 2015) and WHO (2008). After denaturation of the amplicons, $1 \mathrm{ml}$ of the pre-warmed hybridization buffer (HYB) was carefully added to the wells using a pipette and thoroughly mixed. The tray was placed on the TwinCubator ${ }^{\circledR}$ and labeled strips were added to each well ensuring that the strips were completely covered by the liquid and incubated at $45^{\circ} \mathrm{C}$ for 20mins. After incubation, the HYB buffer was aspirated completely from each well and $1 \mathrm{ml}$ of the pre-warmed red stringent wash buffer (STR) was then dispensed into the tray. After 10 minutes incubation at $45{ }^{\circ} \mathrm{C}$ in the TwinCubator $\AA$, STR buffer was aspirated and was washed off with $1 \mathrm{ml}$ of Rinse solution (RIN) for 1 minute. Then $1 \mathrm{ml}$ of the Conjugate (CON) solution was dispensed into each well and incubated for 20 minute on the TwinCubator $\AA$. The strips were washed twice with 1 $\mathrm{ml}$ of Rinse solution (RIN) for 1 minute in the TwinCubator ${ }^{\circledR}$. Then sterile distilled water was added and a 1 minute wash performed on the TwinCubator $\AA$ to wash off the RIN solution after which the distilled water was completely decanted. One (1) $\mathrm{ml}$ of the Substrate solution was then dispensed into each well and incubated for 10 minutes on the TwinCubator ${ }^{\circledR}$ after which the Substrate solution was aspirated and the strips washed twice with sterile distilled water. A pair of clean tweezers was used to remove the strips from the TwinCubator ${ }^{\circledR}$ tray and placed onto absorbent paper. The developed strips were partially dried and transferred to the GenoType $\AA$ MTBDRplus score sheet for interpretation.

\section{RESULTS}

The results of the study revealed that of the 88 sputum samples collected from rifampicin- resistant (confirmed by Genxpert from referring centres) TB patients that were originally on the World Health Organization category I therapythat reported to the Aminu Kano Teaching Hospital, Northwest TB Reference Laboratory, 74 (84.09\%) were males and $14(15.9 \%)$ were females (Table 1$)$. Most of the patients were in the age groups $21-30$ years and 31 40 years. Age group $11-20$ years had the least number of patients (15). Only 1 patient was aged 70 years (Table 1 ).

Table 2 revealed that 86 (97.72\%)of the sputum samples were confirmed as MTBC using theSD BIOLINE TB Ag MPT64 Rapid test while two were invalid.

Results of the GenoType ${ }^{\circledR}$ MTBDRplus molecular line probe assay (LPA) were shown in Table 3, which reveals that of the 88 rifampicin-resistant (by Genexpert) sputum samples, 48 (54.5\%) were identified as MDR-TB, $29(32.9 \%)$ were rifampicin mono-resistant TB cases while only $1(1.36 \%)$ was resistant to isoniazid. Furthermore, 8 sputum samples were found to be susceptible to both isoniazid and rifampicin.

Table 4 describes the pattern of genetic mutations of the rifampicin-resistant samples. According to the study, among the 78 rifampicin-resistant TB samples (i.e., $M D R=48, \mathrm{RIF}=29$ and $\mathrm{INH}=1$ ), detection of rifampicin resistance due to the rhoBmissing WT geneand the presence of rhoBMUT gene was seen in $77(98.7 \%)$ and $51(61.4 \%)$ of them respectively.

Similarly, Table 4 further describes that among the 78 rifampicin-resistant samples, detection of isoniazid resistance due to katG missing WT gene and presence of katG MUT gene was seen equally in 35 (44.9\%) of them.

Among the 78 rifampicin-resistant isolates, resistance due to inhA missing WT gene was observed in only 9 $(11.5 \%)$ of them, whereas resistance due the presence of inhA MUT gene was only seen in 2 (4.17 $\%$ ) of the samples (Table 4) 
Among the 48 MDR-TB samples, detection of rifampicinresistance due to rhoBmissing WT gene was seen in all $(100 \%)$ of them while $34(70.8 \%)$ of them exhibited resistance due to the presence of rhoBMUT gene (Table 4). Similarly detection of isoniazid resistance due to katG missing WT gene and the presence of katG MUT gene was seen in $34(70.8 \%)$ and $35(72.9 \%)$ of the MDR-TB samples respectively.INH resistance in the MDR-TB cases was further associated with InhA WT and InhA MUT in $16.67 \%$ and $4.17 \%$ of the isolates respectively

Table 1: Distribution of age and sex among rifampicin resistantTB patients attending Aminu Kano Teaching Hospital, Northwest TB Reference Laboratory,Kano Nigeria.

\begin{tabular}{|c|c|c|c|}
\hline $\begin{array}{l}\text { AGE } \\
\text { (years) }\end{array}$ & $\begin{array}{l}\text { SEX } \\
\text { MaleFemale } \\
\text { No }(\%)\end{array}$ & No $(\%)$ & Total \\
\hline $11-20$ & 114 & & 15 \\
\hline $21-30$ & 254 & & 29 \\
\hline $31-40$ & 223 & & 25 \\
\hline $41-50$ & 151 & & 16 \\
\hline $51-60$ & 11 & & 2 \\
\hline $61-70$ & 01 & & 1 \\
\hline TOTAL & $74(84.09)$ & $14(15.91)$ & 88 \\
\hline
\end{tabular}

Table 2: Status of AFB Smear Positive Rifampicin-Resistance TB sputum samples from patients attendingAminu Kano Teaching hospital, Northwest TB Reference Laboratory, Kano, Nigeria using SD BIOLINE TB Ag MPT64 Rapid test.

\begin{tabular}{lc}
$\begin{array}{l}\text { Organism identifiedAFB Smear Positive Rifampicin-Resistance Sputum Samples(SD BIOLINE TB Ag } \\
\text { MPT64)Number (\%) }\end{array}$ & $86(97.72)$ \\
\begin{tabular}{lc} 
Mycobacterium tuberculosis complex & $2(2.28)$ \\
Invalid & $\mathbf{8 8}$ \\
\hline Total &
\end{tabular} \\
\hline
\end{tabular}

Table 3: Drug resistance and susceptibility profile of 88 rifampicin-resistant TB patients attending Aminu Kano Teaching Hospital, Northwest TB Reference Laboratory, Kano, Nigeria.

Drug resistance and Susceptibility status

INH resistance

RIF resistance

MDR TB

Susceptible

Invalid
No

\section{Number of isolates $(n=88)$}

$\%$
Among the 29 rifampicin mono-resistant sample, resistance attributed torhoBmissing WT gene was (100\%) samples, whereas 21 presence of rhoBMUT gene (Table 4). And, finally, the only isoniazid mono-resistant isolate among the 29 restance pattern to both inhA missing WT gene respectively.

\begin{tabular}{rc}
1 & 1.36 \\
29 & 32.9 \\
48 & 54.5 \\
8 & 9.0 \\
2 & 2.3 \\
\hline
\end{tabular}


Table 4: Pattern of genetic mutations of MTBC confirmed sputum samples from rifampicin-resistant TB patients at Aminu Kano Teaching hospital, Northwest TB Reference Laboratory, Kano,Nigeria.

\begin{tabular}{|c|c|c|c|c|c|}
\hline \multirow[b]{2}{*}{ Gene } & \multirow[b]{2}{*}{$\begin{array}{l}\text { Band type/ } \\
\text { mutation region }\end{array}$} & \multicolumn{3}{|l|}{ Resistance status } & \multirow[b]{2}{*}{ Total $(n=78)$} \\
\hline & & $\begin{array}{l}\text { MDR } \\
(n=48)\end{array}$ & $\begin{array}{l}\text { RIF-R } \\
(n=29)\end{array}$ & $\begin{array}{l}\text { INH-R } \\
\qquad(n=1)\end{array}$ & \\
\hline \multirow[t]{2}{*}{ rho $B$} & WT missing & $48(100 \%)$ & $29(100 \%)$ & 0 & $77(98.7 \%)$ \\
\hline & MUT present & $30(62.5 \%)$ & $21(72 \%)$ & 0 & $51(61.4 \%)$ \\
\hline \multirow[t]{2}{*}{ kat $G$} & WT missing & $34(70.8 \%)$ & 0 & $1(100 \%)$ & $35(44.9 \%)$ \\
\hline & MUT present & $35(72.9 \%)$ & 0 & 0 & $35(44.9 \%)$ \\
\hline \multirow[t]{2}{*}{$\operatorname{Inh} A$} & WT missing & $8(16.67 \%)$ & 0 & $1(100 \%)$ & $9(11.5 \%)$ \\
\hline & MUT present & $2(4.17)$ & 0 & 0 & $2(2.6 \%)$ \\
\hline
\end{tabular}

\section{DISCUSSION}

The backbone of a successful TB case management depends largely on, accurate and rapid detection of new cases and, prompts and proper monitoring of infected cases to avert the evolution and spread of drug resistant TB and indeed MDR-TB which could lead to a more complicated XDR-TB, thus jeopardizing efforts to control TB. In this regard several rapid diagnostic tests have been evolved including GenoType ${ }^{\circledR}$ MTBDR plusassay which was used in this study to describe the multidrug resistant profile of TB patients initially identified with rifampicin-resistance (by GeneXpert) referred from various centers across the Northwestern zone of Nigeria.

This study revealed that of the 88 initially confirmed rifampicin-resistance patients who were on WHO category I anti-tuberculosis drugs that reported to the Aminu Kano Teaching Hospital, Northwest TB Reference Laboratory, Kano 48(54.5\%) were identified as MDR-TB (by line prove assay) and this was higher than the reports by Qazietal.(2014) and Tripathiet al. (2012) who reported MDR-TB rate of $38 \%$ among resistant cases in Pakistan and $43.37 \%$ in India from patients that were refractory to chemotherapy for $>12$ month respectively. However, the results were similar to $54 \%$ of MDR-TB in Georgia, USA reported by Tukvadzeet al. (2012). Thus, our study identifies that in a setting typified with inadequate provisions of well-organized TB reference laboratories the reported MDR-TB rate of $54 \%$ is rather alarming especially bearing the fact that the WHO (2013a) revealed that the prevalence of MDRTB among previously treated cases was about $20 \%$.

The study revealed that $29(32.9 \%)$ of the TB patients were identified with rifampicin monoresistance.

The 8 susceptible cases and 1 INH mono-resistant case reported in this study were earlier identified as RIF resistance by GeneXpert at their initial healthcare center, however our report detected them as susceptibleand as INH mono resistant respectively by line prove assay. More so, 3 out of the 8 cases were patients that were primarily not on any anti-TB drug but identified as RIF resistance meaning that it could be an acquired drug resistance. So many factors might account for the observed discrepancy. Firstly, the initial report of RIF resistance status of the patients by their respective health facility could be an inappropriate diagnosis, which indicated the need to conduct and report the TB diagnosis with expertise and caution by the appropriate authority lack of which could lead to inappropriate administration of anti-TB drugs which is detrimental to the health of the patient and encourages development and spread of drug resistance, MDR and XDR-TB. Secondly, Barnard et al. (2012)described that LPA results are based on banding patterns detected on a strip following hybridization with PCR products amplified from target DNA in a patient specimen, thus the molecular test may not include probes for all possible mutations. Tripathiet al. (2012) also explained that INH and RIF's resistance in MTB complex (MTC) isolates are mainly based on mutations in a limited number of genes.Thus, in this study there is every possibility that the gene regions responsible for rifampicin resistance of the 8 susceptible cases in our study were not identified by the LPA method used.

Barnard et al. (2012) further elaborated that often, confusion arises if a wild-type band (found in drugsusceptible strains) is missing, but a corresponding mutation band (found in drug-resistant strains) is not present. They identified that this banding pattern is the result of a drug resistance-associated mutation different from the common ones identified by the specific probes on the strip. They further revealed that, there is a slight possibility that the pattern represents a silent mutation, one that does not result in an amino acid change and concluded that this is one of the reasons that mutations observed with molecular methods should be confirmed with a phenotypic assay silent mutation. Qaziet al. (2014) also expounded that five (20\%) MDR-TB isolates in their study lack any mutation in the rifampicin resistance determining regions (RRDR) region of the $r$ rpoBgene, though these isolates were phenotypically resistant to RIF. They indicated that thisdifference might persist due to genotype variations prevailing worldwide. Another important observations made by Barmankulova (2015) was that although Xpert MTB/RIF showed higher accuracy in detecting rifampicin resistance TB cases with a sensitivity and specificity of $96.8 \%$ and $91.8 \%$ respectively in their study, yet they identified that furtherstudies ofXpert MTB/RIF in a general population with better adherence to laboratory protocol and solid recording systems may yield more accurate information.Albert et al. (2010) earlier stressed that careful attention must be paid to training, supervision andadherence to stringent laboratory protocols to ensure high quality results during routine implementation. 
Rifampicin resistance was found out to be associated with transcriptional process that target the mycobacterial RNA polymerase leading to mutations in the $r p o B$ locus, thus conferring conformational changes leading to defective binding of the drug and consequently resistance (Jin and Gross, 1988). Yang et al. (2011) further revealed that $r p o B$ mutations are more likely segregated in the 81-bp regions called the rifampicin resistance determining regions (RRDR). And because up to $90 \%$ ofrifampicin resistance strains carry RRDR mutations within codons 516 (WT4), 526 (MUT2) and 531(WT8) they are termed as hot spots and are being rapidly used to identify RIF resistant isolates. Changes in the codon Ser531 (MUT8) and His526 (MUT2) have been documented in more than $70 \%$ of RIF resistant isolates (Rattan et al. 1988).

This study also illustrated the pattern of genetic mutations of the studied cases, in which resistance due to the missing $r p o B$ WT gene was seen in 77 $(98.7 \%)$ of the 78 rifampicin resistant TB cases and resistance due to the mutations in rpoB MUT gene was observed in $51 / 78(61.4 \%)$ of them. This report is comparable to observations by Qaziet al. (2014) who indicated that more than $90 \%$ of the mutations were reported in the $r h o B$ WT gene region. Similarly, Tukvadzeet al. (2012) revealed that the most common genetic abnormality in their study was associated with lack of binding to the WT8 in $80 \%$ of the isolates, followed by the S531L (MUT3) in $68 \%$. However Barnard et al., (2008) in a study in Cape Town, South Africa reported that detection of rifampicin resistance of $70.5 \%$ was due to mutations of S531L (MUT3).

This study further recorded a100\% rifampicin resistance due tomissing $r p o B$ WT gene among the MDR (48) and rifampicin mono-resistant (29) cases in our study, while Barnard et al. (2008) reported that $76.4 \%$ and $37.5 \%$ of MDR and RIF mono-resistant isolates exhibited resistance due to mutations in $r p o B$ MUT gene region. Our study supports the earlier documented observations that detection of rifampicin resistance could be used as a surrogate marker for development of MDR-TB.

The observations of our study correlate with documented works that reported frequency inresistant strains in respect to rifampicin is attributed to $r p o B$ in $>95 \%$ and that to $k a t G$ was $60-70 \%$ (Rattan et al., 1998) and most katGmutations are found between codons 138 and 328 with the most commonly observed gene alteration at codon 315 (WT) (60 $80 \%$ of cases) (Barnard et al., 2012). Moreover, most frequent mutation at codon 315 is a Ser $\rightarrow$ Thr substitution that is estimated to occur in $30-60 \%$ of all INH-resistant isolates. This study revealed that detection of isoniazid resistance due to katG missing WT gene and katG MUT gene was seen in 35/78 $(44.9 \%)$ of the cases and this was lower than the reports of Barnard et al. (2008) who revealed that of all INH resistant strains $64.1 \%$ had mutations in the kat $G$ gene region. Furthermore, among the 48 MDR cases in this study, INH resistance due to katG missing WT gene was recorded in $34(70.8 \%)$ and this was similar to the reports of Barnard et al. (2008) who also recorded $70.8 \%$ INH resistance in MDR strains to be due to mutations in the $k a t G$ gene.

Efforts to determine factors involved in resistance to INH led to the discovery of inhAlocus, which was proposed as the primary target of coresistance to INH and ethionamide (Rattan et al., 1998). According to Barnard et al. (2012), inhAencodes a mycobacterial enoyl-ACP reductase enzyme that is probably involved in the biosynthesis of mycobacterial cell wall fatty acids. The most common mutations of inhAoccur in the promoter region of the gene and are most frequently seen at positions $-24(\mathrm{~g} \rightarrow \mathrm{t}),-16(\mathrm{a} \rightarrow \mathrm{g})$, or $-8(\mathrm{t} \rightarrow \mathrm{g} / \mathrm{a})$ and $-15(\mathrm{ct} \rightarrow)$.

The prevalence mutations in inhA and katG genes seems to vary widely in different geographic locations (Barnard et al., 2008), strains bearing mutations in the coding region of inhAshow low-level resistance and studies revealed that evidence of mutations in theinhA locus and INH resistance were shown to have about 10\% correlation (Barnard et al., 2012; Rattan et al., 1998). And this corroborated with the findings of our study which revealed that INH resistance due to missing inhA WT gene was reported in $9(11.5 \%)$ TB cases who also exhibited mutation in the kat $G$ gene. Barnard et al. (2008) reported a similar finding of $12(13.48 \%)$ of resistant strains had mutations inkatG and inhA genes regions.Thus, it is interesting to note that INH resistance in some of our studied cases was due not only to mutations in the kat $G$ gene but also to mutations in the inhA gene. The low level of expression of INH resistance due to mutations in theinhA gene in this study might be attributed to the fact that the patients were on category I TB treatment regimens that does include ethionamide and Tukvadzeet al. (2012) revealed thatethionamide inhibitsinhA, thus if inhA mutations are present ethionamide resistance is highly likely.

Specifically, the INH resistance recorded in this study in $8(16.67 \%)$ of the MDR cases were due to missinginhA WT gene in addition to having mutations in the katG gene. However, contrary to the findings of this study Barnard et al. (2008) reported that $27 \%$ of the MDR isolates did not have mutations in the katG gene, but were identified as INH resistant as a result of mutations in the inhA WT gene region only. According toTukvadzeet al. (2012) if mutations are detected in only the inhA gene the isolate likely has low level resistance to INH, thus high dose may have clinical effect.

In conclusion, this study revealed a 54\% MDR-TB rate among rifampicin-resistant TB patients in Northwestern Nigeria.Amongthe MDR-TB casesRIF resistance was associated with $r p O B$ WT gene and rpoB MUT gene in $100 \%$ and $62 \%$ of the isolates, and INH resistance was associated with katG WT gene and katG MUT gene in $70.8 \%$ and $72.9 \%$ of the isolates respectively. INH resistance in the MDR-TB cases was further associated with InhA WT and InhA MUT in $16.67 \%$ and $4.17 \%$ of the isolates respectively. The study also identifies the importance of expertise in the conduct and diagnosis of rifampicin-resistance status of TB patients by the GeneXpert methods lack of which may be detrimental to the health of the patient. 
Thus, the study solicits for a much more committed TB control programmes (including provision and training of manpower) by the relevant agencies and recommends that efforts should be made towards rapid and accurate diagnosis of TB patients, and proper treatment and management of the infected cases to minimize the spread and evolution of MDR-

\section{REFERENCES}

Albert, H.,Bwanga, F.,Mukkada, S.,Nyesiga, B.,Ademun, J.P., Lukyamuzi, G., Haile, M.,Hoffner, S.,Joloba, M. and O'Brien, R. (2010):Rapid screening of MDR-TB using molecular Line Probe Assay is feasible in Uganda. BMC Infectious Diseases, 10 (41): 1-7. http://www.biomedcentral.com/14712334/10/41

Barmankulova A., Higuchi, M., Sarker, M.A., Alim, A. andHamajima, N. (2015): Tuberculosis And Rifampicin Resistance Among Migrants In Kyrgyzstan: Detection By A New Diagnostic Test. Nagoya Journal of Med. Sci. 77: 41-49.

Barnard, M., Parsons, L.,Miotto, P.,Cirillo, D.,Feldmann, K., Gutierrez, C. andSomoskovi, A. (2012): Molecular Detection of DrugResistant Tuberculosis By Line Probe Assay: Laboratory Manual for Resource-Limited Settings. Foundation for Innovative New Diagnostics. www.finddiagnostics.org.

Barnard, M., Albert, H., Coetzee, G., O'Brien, R. andBosman, E.M. (2008): Rapid Molecular Screening for Multidrug-Resistant Tuberculosis in a High-Volume Public Health Laboratory in South Africa. American Journal of Respiratory and Critical Care Medicine, 177: 787-792. www.atsjournals.org

Genotype MTBDRplus VER 2.0 (For LPA); Manufactured by HAINLifeSCIENCE, GermanyMan Date: 2015-08-28; EXP 2016-03

Jin, D. and Gross, C. (1988): Mapping And Sequencing Of Mutations InEscheria colirpoB Gene That Lead To Rifampicin Resistance. Journal of Molecular Biology, 202:45-48

Nathanson, E., Nunn, P., Uplekar, M., Floyd, K., Jaramillo, E., Lönnroth, K., Weil, D. andRaviglione, M. (2010): MDR Tuberculosis Critical Steps for Prevention and Control. New England Journal of Medicine, 363 (11):1050-8.

National Committee for Clinical Laboratory Standards (NCCLS) (2000).Susceptibility of Mycobacteria, Norcadia, and Actinomycetes $2^{\text {nd }}$ ed. Tentative Standards M24-T2 Wayne, P.A.

National Tuberculosis and Leprosy Programme Control (NBTLC) (2011). Manual on Technical SOPs for Tuberculosis Laboratories in Nigeria.

Qazi, O., Rahman, H., Tahir, Z., Qasim, M., Khan, S., Anjum, A.A.,Yaqub, T., Tayyab, M., Ali, N. andFiryal, S. (2014): Mutation pattern in rifampicin resistance determining region of rpoB gene in multidrug-resistant Mycobacterium tuberculosis isolates from
TB and indeed XDR-TB whose treatment is presented with more challenges.

\section{AKNOWLEGEMENT}

The authors acknowledge the management of Aminu Kano Teaching Hospital, Kano, Nigeria for the approval given to conduct the study.

Pakistan. International Journal of Mycobacteriology, 3: 173-177

Rattan, A., Kalia, A. and Ahmad, N. (1998) MultidrugResistant Mycobacterium Tuberculosis. Molecular Perspectives. Emerging Infectious Diseases, 4 (2): 195-209

SD BIOLINE TB Ag MPT64 Rapid testmanufactured bySD STANDARD DIAGNOSTICS, INC 65,Borahagal-ro, Giheung-gu, Yonginsi,Gyeonggi-do, Republic of Korea: Exp; 2016: 12:05.

Tripathi D.K., Srivastava K., Kant, S. andSrivastava, K.K. (2012): Molecular Profiling of Drug Resistant Isolates of Mycobacterium tuberculosis in North India. Advances in Microbiology, 2: 317-326. http://www.SciRP.org/journal/aim

Tukvadze, N., Kempker, R.R., Kalandadze, I., Kurbatova, E., Leonard M.K., Apsindzelashvili, R., Bablishvili, N., Kipiani, M. and Blumberg, H.M. (2012) Use of a Molecular Diagnostic Test in AFB Smear Positive Tuberculosis Suspects Greatly Reduces Time to Detection of Multidrug Resistant Tuberculosis. PLOS ONE7 (2): e31563. doi:10.1371/journal.pone.0031563

WHO (2006):Guidelines For The Programmatic Management Of Drug Resistant Tuberculosis.WHO/HTM/TB/2006.361

WHO Policy Statement(2008): Molecular Line Prove Assay For Rapid Screening Of Patients At Risk Of Multidrug Resistant Tuberculosis (MDRTB).World Health Organization,Geneva. Switzerland.

http://www.who.int/tb/features_archive/policy_state ment.pdf

WHO (2010): Multidrug Resistant And ExtensivelyDrug Resistant TB (M/XDR-TB) 2010 Global Report On Surveillance And Response WHO/HTM/TB/20103. Geneva. World Health Organization, 2010.

WHO (2013a): Multidrug Resistant TB (MDR-TB) 2013 Update. www.who.int/tb/2013.

WHO (2013b): Count Down To 2015. Global Tuberculosis Report 2013 Supplement, World Health Organization. WHO/HTM/TB/2013.1. Geneva. World Health Organization, 2010.

Yang, S., Zhong, M., Zhang, Y. and Wang, Y. (2011): Rapid Detection of rpoBAndkatG Genes from the Sputum of Multidrug-Resistant Mycobacterium tuberculosis by Polymerase Chain Reaction (PCR)-Direct Sequencing Analysis. African Journal of Microbiology Research, 5 (26): 4519-4523. http://www.academicjournals.org/AJMR 


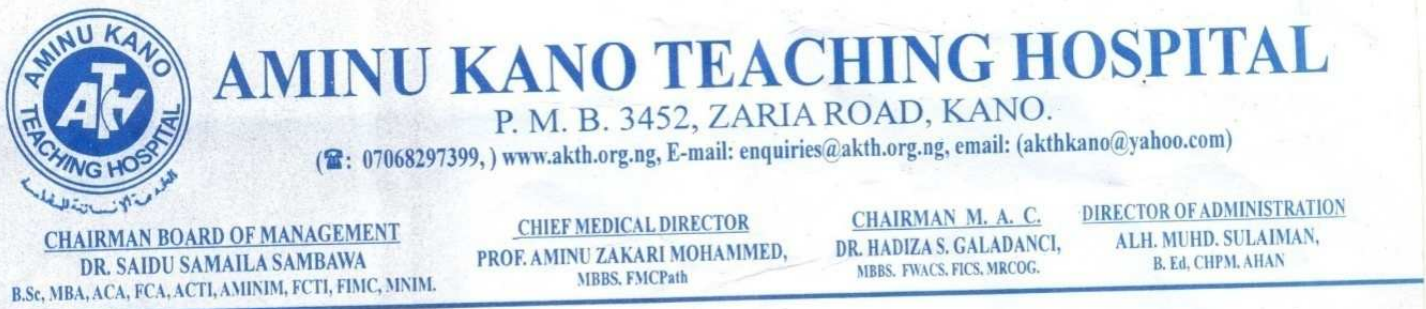

NHREC/21/08/2008/AKTH/EC/1414

AKTH/MAC/SUB/12A/P-3/VI/ 1514

$15^{\text {th }}$ May, 2015

Abubakar Tukur D/Kudu

Department of Microbiology

AKTH, Kano.

Ufs:

The Head of Department

Microbiology

AKTH, Kano.

\section{ETHICS APPROVAL}

Further to your application in respect of your research proposal titled "Drug Susceptibility Profile of Mycobacterium Tuberculosis in Kano North Western Nigeria", the Committee reviewed your proposal and noted same as a Prospective Study.

In view of the above, Ethics approval is hereby granted to conduct the research.

However, the approval is subject to periodic reporting of the progress of the study and its completion to the Research Ethics Committee.

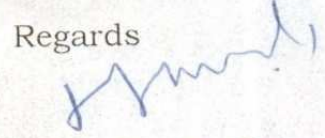

Abubakar S. Mahmud

Secretary, Research Ethics Committee

For: Chairman 